23

\title{
Сдвиговый спекл-интерферометр с квадролинзой
}

\author{
(С) Г.Н. Вишняков ${ }^{1,2}$, В.Л. Минаев ${ }^{1}$, А.Д. Иванов ${ }^{1}$, Ф.Ю. Виноградов ${ }^{1}$ \\ ${ }^{1}$ ФГУП „ВНИИОФИ“, \\ 119361 Москва, Россия \\ ${ }^{2}$ МГТУ им. Н.Э. Баумана, \\ 105005 Москва, Россия \\ ๑ e-mail: acaemi@ya.ru
}

Поступила в редакцию 05.06.2020 г.

В окончательной редакции 05.06.2020 г.

Принята к публикации 23.06.2020 г.

\begin{abstract}
Предложен новый оптический элемент - квадролинза, которая использована в составе сдвигового спекл-интерферометра (шерографа) для обеспечения измерений напряженно-деформированных состояний объектов одновременно в двух взаимно перпендикулярных направлениях. Квадролинза состоит из четырех одинаковых секторов, вырезанных из исходной круглой линзы и разнесенных друг от друга с образованием зазоров. Квадролинза строит четыре изображения объекта, смещенных относительно оптической оси на расстояние, которое зависит от величины зазоров между секторами. Восстановление фазы по одной спеклинтерферограмме проводится методом пространственного фазового сдвига на основе преобразования Фурье. Для повышения контраста интерференционных полос перед секторами квадролинзы устанавливается апертурная диафрагма с четырьмя отверстиями, а для разделения каналов и уменьшения влияния перекрестной интерференции может использоваться поляризационная развязка каналов. Приведены экспериментальные результаты использования спекл-интерферометра с квадролинзой по исследованию микродеформации круглой мембраны.
\end{abstract}

Ключевые слова: сдвиговый спекл-интерферометр, шерограф, квадролинза, дефектоскопия.

DOI: $10.21883 /$ OS.2020.10.50033.170-20

\section{Введение}

Сдвиговый спекл-интерферометр - это устройство, позволяющее на основании рассеянного от шероховатой поверхности объекта когерентного излучения последовательно регистрировать ряд спекл-интерферограмм и после компьютерной обработки получать поле градиента перемещения отдельных его участков. Часто такое устройство называют шерографом (от англ. shear сдвиг). Шерография является одним из современных оптических методов неразрушающего контроля [1] и находит широкое применение, например, в авиационной и космической отрасли.

Для получения информации о поле перемещений в шерографии необходимо осуществить две экспозиции объекта: до воздействия на объект и после воздействия. Воздействия, вызывающие деформацию объекта, могут выполняться различными способами, например механической нагрузкой, тепловым нагревом, изменением давления и т. п.

Современные шерографы используют традиционные для интерферометрии методы компьютерной реконструкции фазы: метод временного фазового сдвига [2] и метод Фурье [3], который иногда называют методом пространственного фазового сдвига.

Метод временного фазового сдвига требует нескольких интерферограмм, за время регистрации которых фаза отраженного от объекта излучения может изме- ниться не только от деформации объекта, но из-за его смещения как целого, вибрации или возмущения окружающей среды. Поэтому метод временного фазового сдвига в основном подходит для измерения смещений со статической нагрузкой.

Динамические измерения можно получить с помощью метода Фурье, который позволяет восстанавливать фазу по одной интерферограмме в полосах конечной ширины, и его можно использовать для неразрушающего контроля с динамической нагрузкой. Поэтому далее будем рассматривать только шерографы, в которых реализуется метод пространственного фазового сдвига по одному кадру.

Основным элементом шерографа является оптическая система, которая создает поперечно-сдвинутые друг относительно друга изображения объекта, интерферирующие между собой при регистрации, создавая спеклинтерферограммы в бесконечно широкой полосе или в полосах конечной ширины (с несущими полосами).

Обычно регулируемый сдвиг изображений осуществляется с помощью интерферометров Майкельсона, реже Маха-Цендера [4]. Наклоном одного из зеркал интерферометров можно формировать два изображения, сдвинутые друг относительно друга. С помощью таких схем можно реализовывать оба метода реконструкции фазы - временного и пространственного фазового сдвига. Наклоном одного из зеркал можно регулировать не только величину сдвига изображений, но и частоту 
полос конечной ширины. Если же второе зеркало интерферометров закрепить на пьезоэлементе, то можно изменять оптическую длину в этом канале и получать различные фазовые сдвиги между интерферирующими пучками.

Другие распространенные методы создания сдвинутых изображений используют такие экзотические оптические элементы, как бипризма Френеля [5] или билинза Бийе [6,7]. С помощью этих методов создаются интерферограммы в полосах конечной ширины и используется метод пространственных сдвигов для реконструкции фазы.

При использовании метода пространственного фазового сдвига для повышения контраста интерференционных полос обычно используется техника диафрагмирования отображающих объективов непрозрачным экраном с одним или несколькими (до четырех) отверстиями [7]. Назовем такой экран апертурной диафрагмой, которая устанавливается перед отображающим объективом. Чаще всего апертурная диафрагма состоит из двух и более отверстий, смещенных относительно центра объектива.

Чувствительность метода шерографии к выявлению дефектов зависит от направления сдвига изображений, так как именно вдоль этого направления визуализируется градиент поля перемещения. Шерография по одному направлению может не распознать некоторые дефекты, ориентированные параллельно направлению сдвига. Для полной характеризации поля деформации объекта необходима информация о градиентах поля перемещений как минимум в двух взаимно перпендикулярных направлениях. Поэтому далее остановимся только на таких типах шерографов, которые позволяют одновременно по одному кадру измерять градиент поля перемещений вдоль, как минимум, двух направлений сдвига.

Динамические измерения деформации одновременно в двух направлениях с помощью метода Фурье приведены в работе [8], в которой использованы два интерферометра Майкельсона, а для освещения объекта используются два лазера с разными длинами волн. Устранение перекрестной интерференции между выходными пучками из двух интерферометров Майкельсона осуществляется с помощью цветового разделения узкополосными светофильтрами.

В другой работе [9] используется схема с двумя интерферометрами Маха-Цендера, а для устранения перекрестной интерференции применяют поляризационное разделение каналов.

В системе, описанной в работе [10], используется многоапертурное диафрагмирование и сдвиг изображений в трех направлениях с помощью трех интерферометров Маха-Цендера. В каждом интерферометре находится свой объектив с круглой диафрагмой, расположенных так, что на выходе они образуют апертурную диафрагму, состоящую из трех отверстий с центрами в вершинах равностороннего треугольника. Направление и величина сдвига изображений контролируются соответствующим наклоном трех светоделительных кубиков.

Недостаток всех этих систем состоит в сложности юстировки и согласования нескольких интерферометров Майкельсона или Маха-Цендера, а также в использовании большого количества оптических элементов и лазеров.

Наиболее перспективная схема шерографа, по нашему мнению, представлена в работе [11]. В этой работе идея использования бипризмы Френеля получила дальнейшее развитие, и для создания сдвига изображений по трем направлениям используются три стеклянных клина, установленные перед отображающим объективом и апертурная диафрагма из трех отверстий по центру каждого клина. Недостаток такой схемы состоит в невозможности менять величину сдвига изображений, так как она определяется углом при вершине клина, а также достаточно сложной системой крепления и юстировки трех клиньев.

Целью настоящей работы является создание простого оптического элемента для получения регулируемого сдвига изображений в двух взаимно перпендикулярных направлениях и создание на его базе макета шерографа с реконструкцией фазы по одному кадру методом пространственного фазового сдвига.

\section{Описание установки и эксперимент}

Развивая идею использования билинзы в шерографии, мы предлагаем новый тип разрезной линзы, которую назвали по количеству составляющих ее элементов квадролинзой. Квадролинза представляет собой четыре одинаковых сектора, вырезанных из исходной круглой линзы с центром $O$, разнесенных друг от друга с образованием равномерных зазоров 7 величиной $2 \Delta$ (рис. 1), параллельных осям симметрии $X$ и $Y$.

На рис. 1 буквами $O_{1}, O_{2}, O_{3}$ и $O_{4}$ отмечены новые положения оптических осей секторов квадролинзы. У каждого сектора есть свой непрозрачный экран с

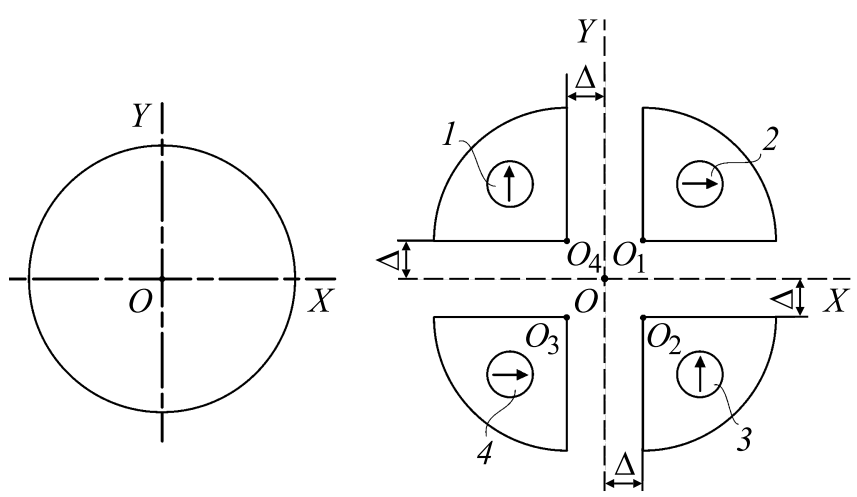

Рис. 1. Чертеж исходной линзы (слева) и квадролинзы (справа). Цифрами указаны номера отверстий апертурной диафрагмы. 


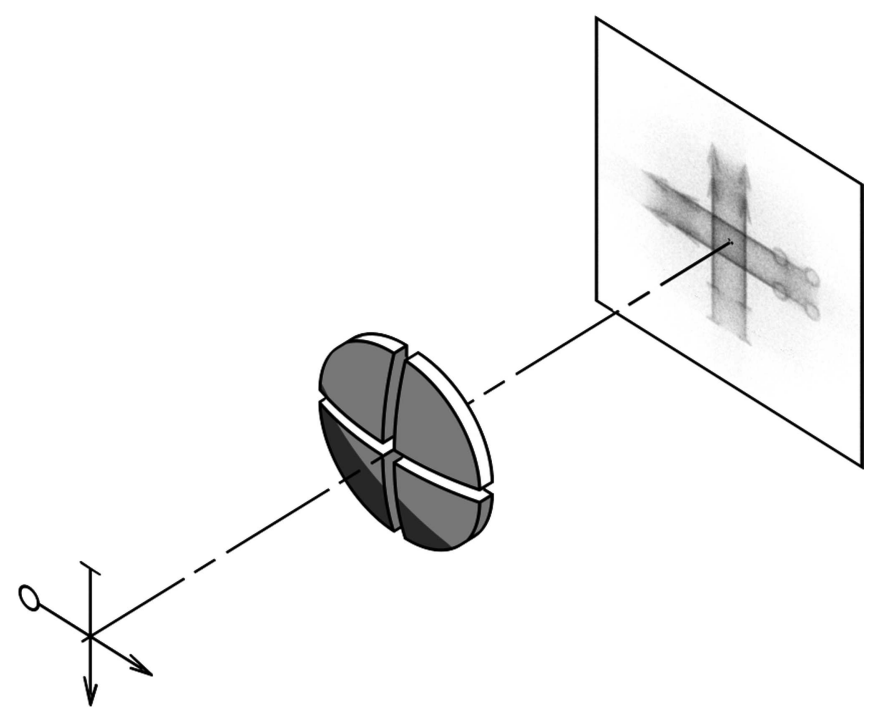

Рис. 2. Изображение, построенное квадролинзой.

отверстием, совокупность которых образует апертурную диафрагму. Внутри отверстий стрелками показаны положения осей скрещенных поляризаторов. Каждый из четырех секторов квадролинзы строит сфокусированное изображение объекта в одной единой для всех секторов плоскости. Эти изображения будут смещены друг относительно друга в плоскости изображений изза того, что оптические оси секторов квадролинзы смещены относительно оптической оси исходной линзы (рис. 2).

Так как эти смещенные изображения образованы делением одного волнового фронта излучения, отраженного от объекта, то они создают сдвиговые спеклинтерферограммы, полосы которых имеют равную частоту и разную ориентацию. Период полос определяется расстоянием между центрами отверстий в апертурной диафрагме, а ориентация полос перпендикулярна прямой, соединяющей центры отверстий в апертурной диафрагме. Как пример, на рис. 4 показана сдвиговая спекл-интерферограмма, которая формируется различными секторами квадролинзы с соответствующими отверстиями в апертурной диафрагме

Для устранения перекрестной интерференции можно использовать поляризационную развязку, как показано на рис. 1. В этом случае будет формироваться только две системы полос, соответствующие отверстиям 1,3 и 2, 4 . Направления сдвига изображений для этих секторов квадролинзы будут перпендикулярны, что и требуется для получения градиентов перемещений в двух перпендикулярных направлениях. Применение поляризаторов позволяет повысить контраст интерференционных полос.

Таким образом, использование квадролинзы в составе ширографа позволяет производить сдвиг изображений в четырех направлениях, избежать дефокусировки и применить метод пространственного фазового сдвига с использованием преобразования Фурье. Изменяя величину зазора $\Delta$ между секторами квадролинзы, можно регулировать величину сдвига изображений, а меняя расстояние между центрами отверстий в апертурной диафрагме, можно подбирать нужную частоту несущих полос в спекл-интерферограмме.

Применение цифрового фурье-преобразования к суммарной сдвиговой спекл-интерферограмме позволяет выделять различные порядки в ее спектре и восстанавливать фазовые картины для четырех различных направлений сдвига. Для восстановления фазовой картины методом пространственного фазового сдвига [3] выполняется пространственная фильтрация суммарного спектра полосовым фильтром, центр которого совпадает с максимумом фурье-спектра в одном из порядков. Суммарная спекл-интерферограмма имеет сложный пространственный вид и должна иметь больший динамический диапазон по сравнению с одиночной интерферограммой. Поэтому желательно применять схему с поляризаторами, в которой регистрируются всего две интерферограммы.

На рис. 3 приведена схема экспериментальной установки, которая использовалась для проверки работы шерографа с квадролинзой. В качестве тест-объекта для исследования выступала деформируемая по центру круглая мембрана 3 диаметром $100 \mathrm{~mm}$, которая ранее использовалась в качестве меры для калибровки спеклинтерферометра [12]. Мембрану освещал лазер $1 \mathrm{c}$ длиной волны $535 \mathrm{~nm}$. Изображение мембраны строилось на матрице видеокамеры 7 посредством оптической системы, состоящей из квадролинзы и апертурной диафрагмы с четырьмя отверстиями, которые могли отдельно закрываться. Квадролинза была изготовлена

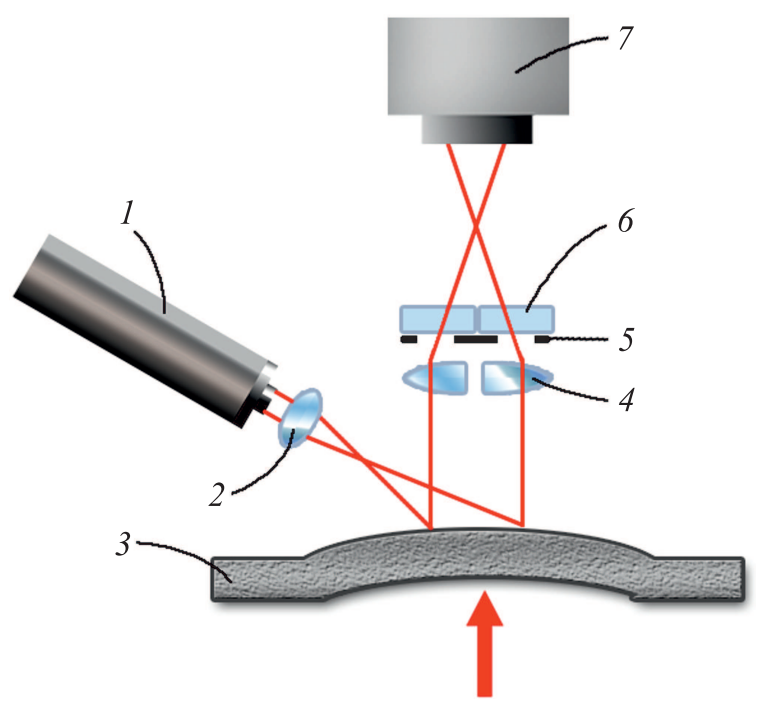

Рис. 3. Схема экспериментальной установки. Стрелкой показано направление механического усилия для деформации мембраны. 1 - лазер, 2 - линза, 3 - мембрана, 4 квадролинза, 5 - апертурная диафрагма, $6-$ поляризаторы, 7 - видеокамера. 


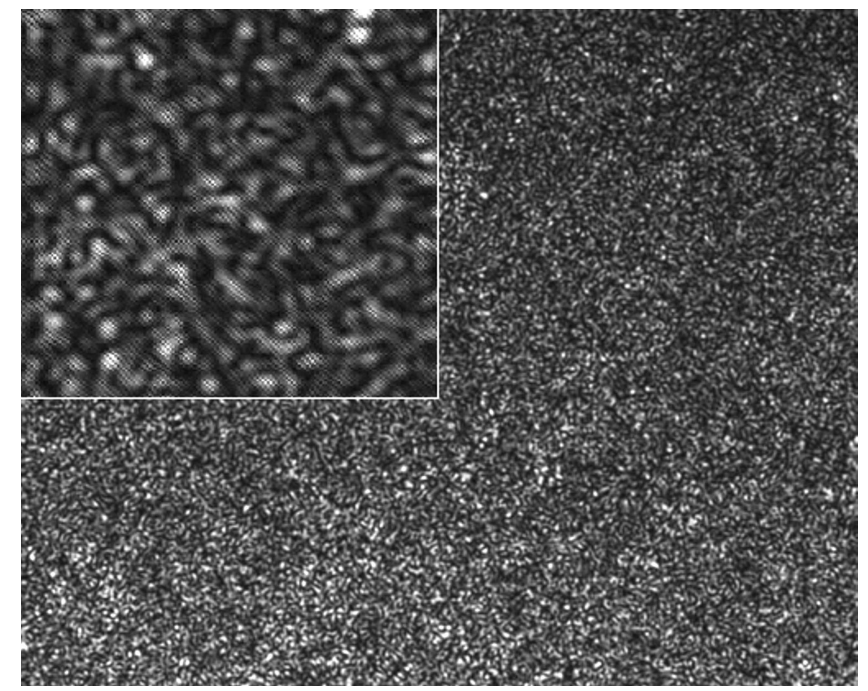

Pис. 4. Спекл-интерферограмма тест-объекта. Во врезке показано увеличенное изображение спекл-структуры, промодулированное системой из четырех высокочастотных интерференционных полос.

из плоско-выпуклой линзы диаметром $52 \mathrm{~mm}$, фокусное расстояние $120 \mathrm{~mm}$ фирмы Thorlabs, путем разреза еe в двух перпендикулярных направлениях, проходящих через центр линзы. Квадролинза располагалась на расстоянии $300 \mathrm{~mm}$ от поверхности мембраны и закреплена в пластиковую оправу, изготовленную на 3D принтере Formlabs Form 2.

Сдвиг секторов квадролинзы относительно ее оптической оси составил $2 \Delta=2 \mathrm{~mm}$ в двух направлениях. Поле зрения камеры на объекте составило $10 \mathrm{~mm}$. В первых экспериментах поляризационная развязка из элементов 6 не применялась, а вместо нее закрывались и открывались соответствующие отверстия в апертурной диафрагме.

В результате экспериментов были получены две спекл-интерферограммы: до и после деформации мембраны в различных режимах работы квадролинзы. Спекл-интерферограммы представляли собой сумму четырех смещенных спекл-изображений объекта, промодулированных системой высокочастотных интерференционных полос в четырех различных направлениях (рис. 4).

В среднем на каждый спекл приходится около 7 полос. Ширина полосы составляет примерно 1 пиксель изображения.

Была проведена серия экспериментов, где каждый раз мембрана деформировалась на $10 \pm 1 \mu \mathrm{m}$, и были получены спекл-интерферограммы объекта до и после деформации, а также их фурье-спектры при различных вариантах открытия отверстий в апертурной дифрагме. На рис. 5 представлены двухмерные спектры, полученные в экспериментах (яркость изображений увеличена).

Из спектров можно видеть, как порядки дифракции на рис. 5, $a, b$ расходятся по диагонали. На рис. 5, появляются дополнительные порядки дифракции от интерференции между всеми отверстиями апертурной диафрагмы квадролинзы: по вертикали, горизонтали и диагоналям.

Следующим шагом обработки интерферограмм по методу пространственного фазового сдвига является выделение области спектра вокруг выбранного порядка дифракции. В настоящей работе использовалась круглая маска, аподизированная двумерным фильтром Баттерворта (параметры фильтра: степень 2, радиус 400 пикселей). После обратного двумерного преобразования Фурье вычислялась фаза полученного поля комплексных чисел. Эта операция применялась для спекл-интерферограмм объекта до и после деформации. Перемножение комплексно сопряженных фаз ведет к устранению вклада в фазу от несущей пространственной частоты и восстановлению разности фаз волновых полей, отраженных от объекта до и после его деформации, в интервале $[0,2 \pi]$ радиан. Эта разностная фазовая картина называется „несшитой“ фазой. Для сглаживания спекл-структуры на „несшитой“ фазе, но с оставлением резких скачков при разрыве фазы также применялась синус-косинусная фильтрация с усреднением до 20 итераций [13].

Полученные поля „несшитой“ фазы, восстановленные от спектров на рис. 5, $a, b$, приведены на рис. 6. Эти картины показывают градиенты полей перемещений в двух взаимно перпендикулярных направлениях.

На рис. 7 приведены варианты восстановления „несшитой“ фазы из суммарного спектра (рис. 5,c), когда отверстия апертурной диафрагмы квадролинзы были открыты. Заметим, что, как и должно быть, фазовые картины, восстановленные из симметричных порядков дифракции, сдвинуты на $\pi$ радиан.
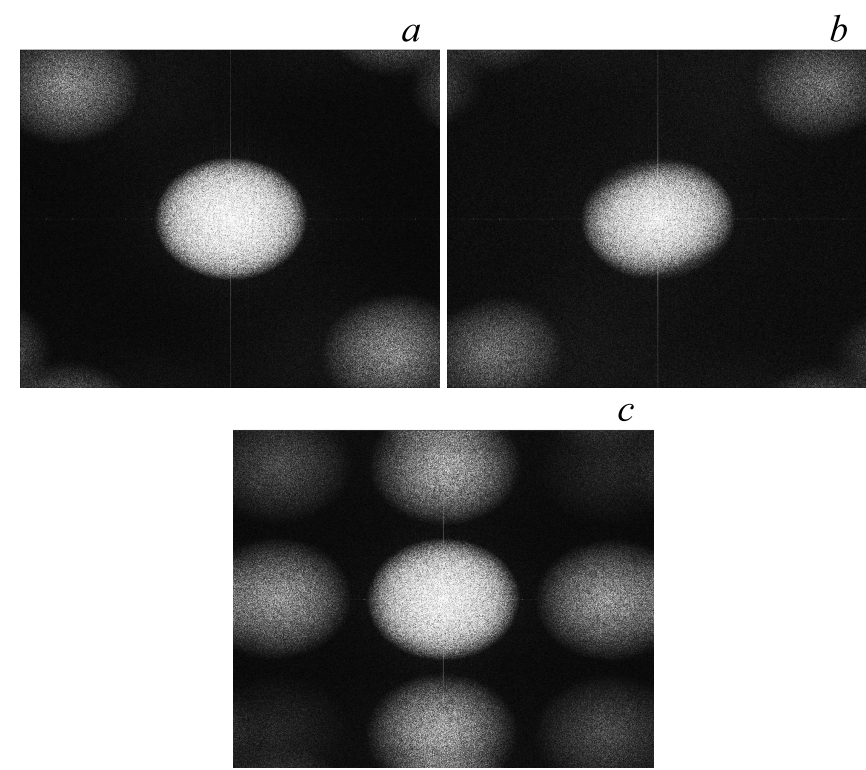

Рис. 5. Двумерные фурье-спектры экспериментальных интерферограмм: $a$ - открыты отверстия $(1,3)$ (рис. 3 ); $b-$ открыты отверстия $(2,4) ; c-$ открыты все отверстия в апертурной диафрагме. 

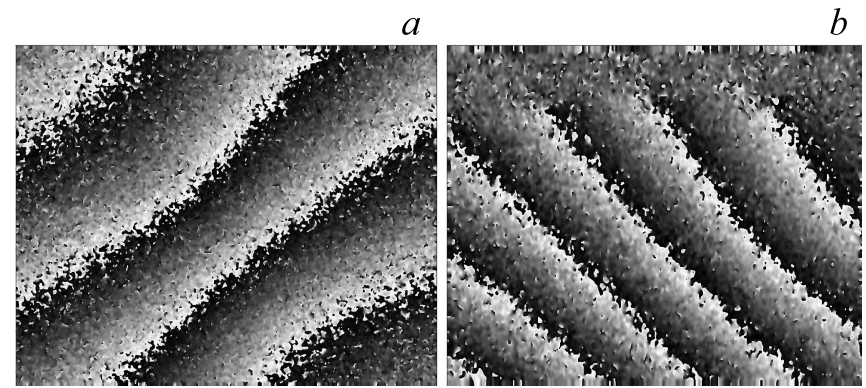

Рис. 6. „Несшитая“ фаза, полученная из спектров на рис. $5, a, b$ соответственно.

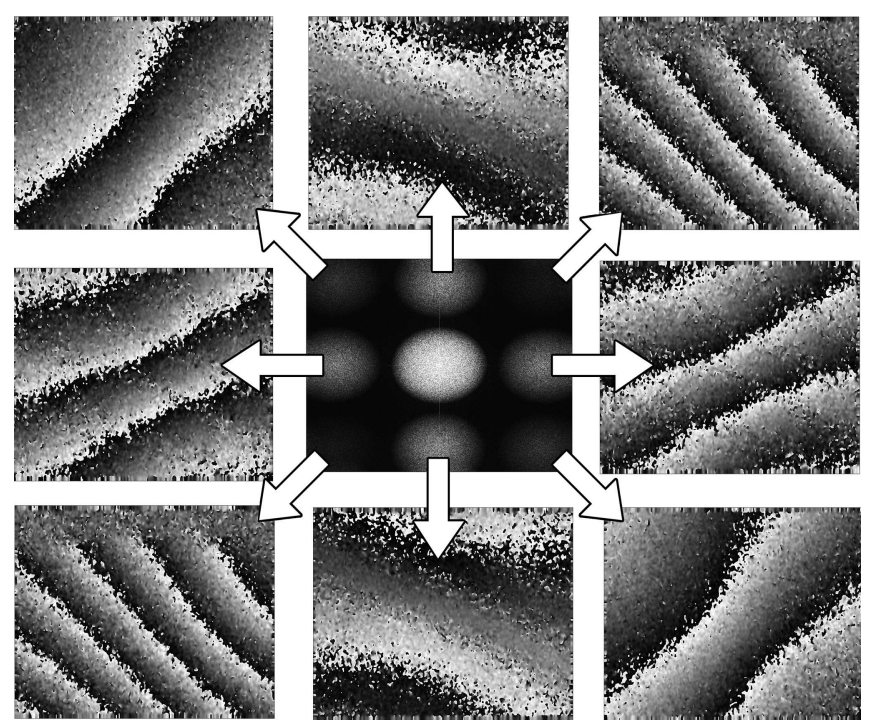

Рис. 7. Картины „несшитой“ фазы, полученные в результате фильтрации спектра (средний рисунок) в различных направлениях.

Таким образом, использовав квадролинзу для разделения каналов, можно получить производную от деформации объекта в четырех направлениях всего по двум спекл-интерферограммам до и после деформации объекта. Более контрастную картину можно достичь устранением перекрестной интерференции, например, с помощью поляризационной развязки с использованием поляризаторов, как на рис. 1.

\section{Выводы}

Разработана и опробована новая конструкция шерографа с использованием квадролинзы. Эксперименты показали возможность получения фазовых изображений в разных направлениях сдвига. Таким образом, благодаря модифицированной сдвиговой оптике предложенный шерограф позволяет расширить спектр обнаруживаемых дефектов под поверхностью различных объектов.

Использование в конструкции шерографа квадролинзы позволяет за одну экспозицию получить спекл- интерферограмму, содержащую информацию о градиенте деформации в двух направлениях, что в свою очередь открывает возможность использовать метод шерографии с динамическими объектами. Кроме того, посредством изменения зазора между секторами квадролинзы можно легко изменять чувствительность шерографа к деформации объекта. Повышение стабильности и снижение требований к юстировке шерографа достигается за счет того, что в нем интерферируют пучки, образованные из одного объектного пучка делением его по волновому фронту на четыре части с помощью секторов квадролинзы, а сдвинутые изображения строятся в фокальной плоскости квадролинзы. Для уменьшения влияния перекрестной интерференции и упрощения вычислений методом пространственного фазового сдвига может быть использовано дополнительное разделение каналов. Для этого перед апертурной диафрагмой следует устанавливать дополнительные элементы, например, линейные поляризаторы.

\section{Финансирование работы}

Работа выполнена с использованием оборудования ЦКП высокоточных измерительных технологий в области фотоники (ckp.vniiofi.ru), созданного на базе ФГУП „ВНИИОФИ“ и поддержанного Минобрнауки России в рамках выполнения соглашения № 05.595.21.0005 (уникальный идентификатор RFMEFI59519X0005).

\section{Конфликт интересов}

Авторы заявляют, что у них нет конфликта интересов.

\section{Список литературы}

[1] Steinchen $W$., Yang L. Digital Shearography: Theory and Application of Digital Speckle Pattern Shearing Interferometry. USA. 2003. 330 p.

[2] James C. Wyant, Phase-Shifting Interferometry. https://wp.optics.arizona.edu/jcwyant/wpcontent/uploads/sites/ 13/2016/08/Phase-Shifting-Interferometry.nb_.pdf

[3] Takeda M., Ina H., Kobayashi S. // J. Opt. Soc. Am. 1982. V. 72. N 1. P. 156. doi 10.1364/JOSA.72.000156

[4] Zhao Q., Dan X., Sun F., Wang Y., Wu S., Yang L. // Appl. Sci. 2018. V. 8. P. 2662. doi 10.3390/app8122662

[5] Nakdate S., Yatagi T., Saito H. // Appl. Optics. 1980. V. 19. N 24. P. 4241. doi 10.1364/AO.19.004241

[6] Murthy R.K., Sirohi R.S, Kothiyal M.P. // Appl. Optics. 1982. V. 21. N 16. P. 2865. doi 10.1364/AO.21.2865_1

[7] Власов Н.Г., Пресняков Ю.П. // Квант. электрон. 1973. T. 14. № 2. C. 80.

[8] Wang Y., Gao X., Xie X., Wu S., Liu Y., Yang L. // Opt. Lasers Eng. 2016. V. 87. P. 197. doi 10.1016/j.optlaseng.2015.12.009

[9] Xie X., Lee C., Li J., Zhang B., Yang L. // Rev. Sci. Instrum. 2016. V. 87(8). P. 083110. doi 10.1063/1.4961473

[10] Peizheng Y., Fangyuan S., Xizuo D., Qihan Z., Yonghong $W$., $Y u$ L. // Optical Engineering. 2019. V. 58(5). P. 054105. doi 10.1117/1.OE.58.5.054105 
[11] Barrera E.S., Analucia F., Willemann P., Benedet D.M., Armando J. // Opt. Lasers Eng. 2018. V. 111. P. 86. doi 10.1016/j.optlaseng.2018.07.018

[12] Ivanov A.D., Minaev V.L., Vishnyakov G.N., Levin G.G. // Meas Tech. 2019. V. 62. P. 95. doi 10.1007/s11018-019-01697-9

[13] Jacquot $P$. Interferometry in Speckle Light: Theory and Applications. 2000. P. 144. doi 10.1007/978-3-642-57323-1 